Technological University Dublin

ARROW@TU Dublin

\title{
A Comprehensive Analysis Verified by Experiment of a Refractometer Based on an SMF28-Small-Core Singlemode Fiber (SCSMF)-SMF28 Fiber Structure
}

\author{
Qiang wu \\ Technological University Dublin, qiang.wu@tudublin.ie \\ Yuliya Semenova \\ Technological University Dublin, yuliya.semenova@tudublin.ie \\ Pengfei Wang \\ Technological University Dublin, pengfei.wang@tudublin.ie
}

See next page for additional authors

Follow this and additional works at: https://arrow.tudublin.ie/engscheceart

\section{Recommended Citation}

Qiang Wu, Yuliya Semenova, Pengfei Wang, Gerald Farrell, "A comprehensive analysis verified by experiment of a refractometer based on an SMF28- Small-Core Singlemode fiber (SCSMF) -SMF28 fiber structure", Journal of Optics, vol. 13, no. 12, 125401, 2011. doi:10.1088/2040-8978/13/12/125401/meta

This Article is brought to you for free and open access by the School of Electrical and Electronic Engineering at ARROW@TU Dublin. It has been accepted for inclusion in Articles by an authorized administrator of ARROW@TU Dublin. For more information, please contact arrow.admin@tudublin.ie, aisling.coyne@tudublin.ie, gerard.connolly@tudublin.ie.

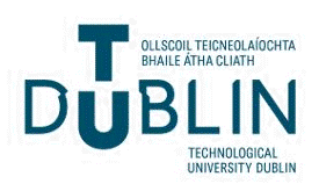


Authors

Qiang wu, Yuliya Semenova, Pengfei Wang, and Gerald Farrell

This article is available at ARROW@TU Dublin: https://arrow.tudublin.ie/engscheceart/156 


\title{
A comprehensive analysis verified by experiment of a
}

\section{refractometer based on an SMF28- Small-Core Singlemode fiber (SCSMF) -SMF28 fiber structure}

\author{
Qiang Wu, Yuliya Semenova, Pengfei Wang, Gerald Farrell \\ Photonics Research Center, School of Electronic and Communications Engineering, Dublin Institute of \\ Technology, Kevin Street, Dublin 8, Ireland (e-mail: qiang.wu@dit.ie).
}

\begin{abstract}
A comprehensive theoretical model for an SMF28- Small-Core SMF (SCSMF) -SMF28 structure based refractometer is developed based on modal propagation analysis (MPA) method. Simulation result shows that the wavelength shift of this refractometer obeys changes exponentially as the surrounding refractive index (SRI) varies. The core diameter of SCSMF does not have a significant influence on the sensitivity of the refractometer but cladding diameter does have. The simulation results are verified experimentally and it is also experimentally demonstrated that a maximum sensitivity of 1808 $\mathrm{nm} / \mathrm{RIU}$ (refractive index unit) for an SRI range from 1.324 to 1.431 and that as expected the wavelength shift response is an exponential function of SRI.
\end{abstract}

Index Terms—Singlemode-multimode-singlemode fiber, refractometer, optical sensing

\section{INTRODUCTION:}

Optical fiber based refractive index (RI) sensors have advantages of high sensitivity, fast response, small size, immunity to electromagnetic interference, remote operation capability, etc. A number of techniques used to implement RI sensing have been reported, such as a fiber Bragg grating (FBG) [1-3], long period grating (LPG) [4-6], surface plasmon resonance [7-9], tapered fiber [10-12] and a singlemode-multimode-singlemode (SMS) fiber structure [13]. Compared to other sensing techniques, an SMS fiber structure based optical sensor has the advantages of low cost and ease of fabrication. It is well 
known that for an SMS fiber structure there is multimode interference within the multimode fiber (MMF) section when light is injected from the singlemode fibre (SMF) into the MMF and that the interference can be influenced by external perturbations such as temperature and strain [14-20]. Our recent investigations show that an etched SMS fiber structure can act as an RI sensor that has experimentally demonstrated an estimated maximum sensitivity of $1815 \mathrm{~nm} / \mathrm{RIU}$ [13]. This indicates that a refractometer based on an SMS fiber structure is a promising technology. However in order to fabricate such an SMS fiber structure based refractometer, the cladding of an MMF must be etched away. This is a disadvantage as the chemical etching process is dangerous and requires a range of safety precautions. Furthermore control of the etching process is complex. More importantly it is very difficult to control the etching process to achieve a repeatable fiber diameter and a smooth fiber surface, both of which will significantly influence the transmission behavior of the etched fiber and hence the performance of the SMS fiber structure based refractive index sensor. An alternative approach is to use a small core singlemode fiber (SCSMF) as a substitute for the etched MMF to construct an SMF28-SCSMF-SMF28 structure based refractometer which was reported recently [21-22]. By depositing a humidity sensing material on the SCSMF, this structure can also be used as a humidity sensor [22-23]. The advantage of using SCSMF is that it is manufactured by conventional means which can guarantee the accuracy of the fiber diameter and smoothness of fiber surface, yielding a simpler and more repeatable SMF28-SCSMF-SMF28 structure based refractometer. There is only limited theoretical analysis in [21-22] which qualitatively predicts the response of the refractometer based on the assumption that the interference takes place between the core mode and cladding modes. However that assumption is not accurate because in theory there is no core mode guided in such a SCSMF as illustrated in [24-25] and our experimental results have verified this. Furthermore in [21-22] there is no quantitative prediction of the spectral shift vs. SRI. In this paper a comprehensive theoretical model for the SMF28-SCSMF-SMF28 structure is provided for the first time based on a mode propagation analysis (MPA) method and simulations are carried out to investigate the influence of the core and cladding diameters of the SCSMF on the sensitivity of the refractometer. Experimental investigations are also carried out to verify the theoretical analysis, demonstrating a maximum RI sensitivity of $1808 \mathrm{~nm} /$ RIU which agrees well with simulation results.

\section{THEORETICAL ANALYSIS}

The configuration of an SMF28-SCSMF-SMF28 structure based refractometer is shown in figure 1. 


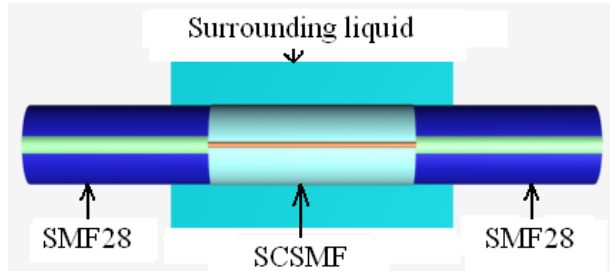

(a)

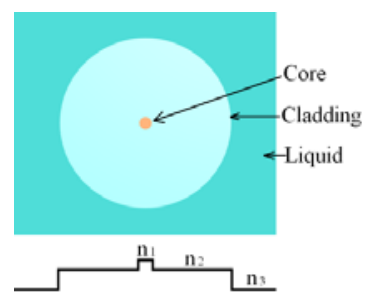

(b)

Fig. 1 Configuration of an SMF28-SCSMF-SMF28 refractometer: (a) view along the fiber axis and (b) cross-sectional view of the SCSMF section

In figure 1, both the SMF28 and SCSMF fibers have a step index profile and the conventional SMF28 fiber is used at both ends as the attaching fiber to the small core SMF fiber. The SCSMF effectively has three layers, namely the fiber core, fiber cladding and surrounding liquid layer. When the refractive index (RI) of the surrounding liquid $\left(\mathrm{n}_{3}\right)$ is less than that of the cladding $\left(\mathrm{n}_{2}\right)$, the light injected from the SMF28 into the SCSMF will excite cladding modes propagating within the cladding of the SCSMF. Multimode interference occurring within the cladding of the SCSMF will dictate the spectral response to the output SMF28, which in turn is affected by the surrounding RI (SRI) of the liquid. By monitoring the spectral changes of the output of this SMF28-SCSMF-SMF28 structure, the SRI can be determined.

To theoretically analyze the operation of the SMF28-SCSMF-SMF28 refractometer, firstly we assume that the SMF28 and SCSMF are ideally aligned. In this case due to the circular symmetry of the input field, only zero order azimuthal modes will be excited in the SCSMF when light is injected from the SMF28 to the SCSMF. Assuming the mode propagation constant within SCSMF as $\beta$ and field profile within SCSMF as $\Psi(r)$, the input field at the SCSMF can be written as [26-27]:

$$
\Psi(r)=\left\{\begin{array}{cl}
A_{0} J_{0}\left(u \frac{r}{a}\right) & r \leq a \\
A_{1} J_{0}\left(u^{\prime} \frac{r}{b}\right)+A_{2} Y_{0}\left(u^{\prime} \frac{r}{b}\right) & a \leq r \leq b \\
A_{3} K_{0}\left(v \frac{r}{b}\right) & r \geq b
\end{array} \quad k_{0} n_{3}<\beta<k_{0} n_{2}\right.
$$


and as

$\Psi(r)=\left\{\begin{array}{cc}A_{0}^{\prime} J_{0}\left(u \frac{r}{a}\right) & r \leq a \\ A_{1}^{\prime} I_{0}\left(v^{\prime} \frac{r}{b}\right)+A_{2}^{\prime} K_{0}\left(v^{\prime} \frac{r}{b}\right) & a \leq r \leq b \\ A_{3}^{\prime} K_{0}\left(v \frac{r}{b}\right) & r \geq b\end{array} \quad k_{0} n_{2}<\beta<k_{0} n_{1}\right.$

where $J_{0}, Y_{0}, I_{0}$ and $K_{0}$ are zero order usual Bessel and modified Bessel functions, $a$ and $b$ are the radii of fiber core and cladding, $\mathrm{n}_{1}, \mathrm{n}_{2}$ and $\mathrm{n}_{3}$ are the RI values of the fiber core, cladding and surrounding liquid respectively, $A_{0}, A_{0}{ }^{\prime}$ are the normalization coefficients, and $u, u^{\prime}, v, v^{\prime}, A_{1}, A_{1}^{\prime}, A_{2}, A_{2}^{\prime}, A_{3}, A_{3}{ }^{\prime}$ are defined as follows:

$$
\left\{\begin{array}{l}
u=a \sqrt{k_{0}^{2} n_{1}^{2}-\beta^{2}} \\
u^{\prime}=b \sqrt{k_{0}^{2} n_{2}^{2}-\beta^{2}} \\
v=b \sqrt{\beta^{2}-k_{0}^{2} n_{2}^{2}} \\
v^{\prime}=b \sqrt{\beta^{2}-k_{0}^{2} n_{3}^{2}}
\end{array}\right.
$$

$$
\begin{aligned}
& \left\{\begin{array}{l}
A_{1}=\frac{\pi A_{0}}{2}\left[u J_{1}(u) Y_{0}\left(u^{\prime} c\right)-u^{\prime} c J_{0}(u) Y_{1}\left(u^{\prime} c\right)\right] \\
A_{2}=\frac{\pi A_{0}}{2}\left[u^{\prime} c J_{1}\left(u^{\prime} c\right) J_{0}(u)-u J_{1}(u) J_{0}\left(u^{\prime} c\right)\right] \\
A_{3}=\frac{1}{K_{0}(v)}\left[A_{1} J_{0}\left(u^{\prime}\right)+A_{2} Y_{0}\left(u^{\prime}\right)\right]
\end{array}\right. \\
& \left\{\begin{array}{l}
A_{1}^{\prime}=A_{0}^{\prime}\left[v^{\prime} c J_{0}(u) K_{1}\left(v^{\prime} c\right)-u J_{1}(u) K_{0}\left(v^{\prime} c\right)\right] \\
A_{2}^{\prime}=A_{0}^{\prime}\left[v^{\prime} c J_{0}(u) I_{1}\left(v^{\prime} c\right)+u J_{1}(u) I_{0}\left(v^{\prime} c\right)\right] \\
A_{3}^{\prime}=\frac{1}{K_{0}(v)}\left[A_{1}^{\prime} I_{0}\left(v^{\prime}\right)+A_{2}^{\prime} K_{0}\left(v^{\prime}\right)\right]
\end{array}\right.
\end{aligned}
$$

where $k_{0}=2 \pi / \lambda, c=a / b, \lambda$ is the wavelength of light in the vacuum. Assuming the light in the input SMF28 has a fundamental mode field distribution $E(r, 0)$ and the field profile within SCSMF is $\Psi(r)$, the input field at the SCSMF can be written as

$$
\begin{aligned}
& \Psi(r)=\sum_{m=1}^{M} \Psi_{m}(r) \\
& E(r, 0)=\sum_{m=1}^{M} b_{m} \Psi_{m}(r)
\end{aligned}
$$

where $E(r, 0)$ is the eigenmode of the SMF28, $\Psi_{m}(r)$ is $m$-the eigenmode of the step index optical fiber SCSMF, which was given by (1) and (2), $M$ is the total number of modes supported in the SCSMF and $b_{m}$ is the excitation coefficient for each mode, which can be expressed as: 
$b_{m}=\frac{\int_{0}^{\infty} E(r, 0) \Psi_{m}(r) r d r}{\int_{0}^{\infty} E(r, 0) E(r, 0) r d r}$

The field within the SCSMF section at a propagation distance $z$ can thus be calculated by

$$
E(r, z)=\sum_{m=1}^{M} b_{m} \Psi_{m}(r) \exp \left(j \beta_{m} z\right)
$$

where $\beta_{m}$ is the propagation constant of each eigenmode within the SCSMF. The transmitted power to the output SMF28 thus can be expressed as

$$
P_{\text {out }}(z)=\frac{\left|\int_{0}^{\infty} E(r, z) E(r, 0) r d r\right|^{2}}{\int_{0}^{\infty}|E(r, z)|^{2} r d r \int_{0}^{\infty}|E(r, 0)|^{2} r d r}
$$

As the SRI changes, the propagation constant of the cladding mode will change and hence the excited eigenmodes in the cladding of the SCSMF will change, resulting in changes to the excitation coefficient of each mode $b_{m}$ in Eq. (8), the interference within the SCSMF in Eq. (9) and the output to the SMF28 in Eq. (10). If the core diameter of the SCSMF is very small compared to that of SMF28, it is possible that the $V$ parameter is less than $1\left(V=\frac{2 \pi a}{\lambda} \sqrt{n_{1}^{2}-n_{2}^{2}}<1\right)$ and hence there is no guided core mode within the SCSMF [24-25].

However cladding modes are excited within the SCSMF. Figure 2 shows the simulated $1^{\text {st }}$ and $15^{\text {th }}$ order cladding modes within SCSMF to illustrate that multimode propagation is taking place in the SCSMF. In this simulation, the surrounding liquid has RI of 1.32 and the wavelength is $1500 \mathrm{~nm}$, the SCSMF has core and cladding diameters of 2.2 and $125 \mu \mathrm{m}$ respectively and the refractive indices of the core and cladding are 1.451 and 1.445 respectively. Figure 2(b) shows that the general cladding mode has a ring like structure across the whole cross section of the cladding region, which agrees with the previous report in [28]. With those parameters, if the wavelength is longer than $1500 \mathrm{~nm}$, the $V$ value will be less than 0.61 , which means that there is no guided core mode in the SCSMF [24-25]. 


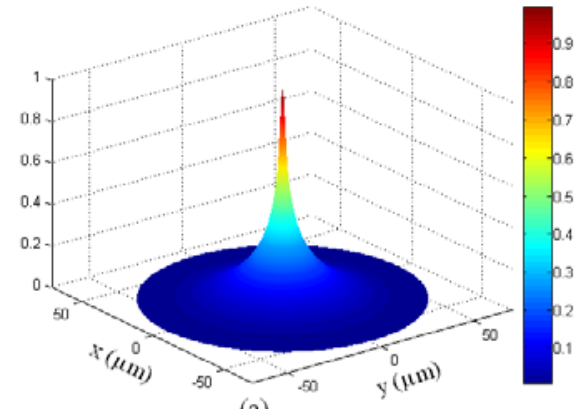

(a)

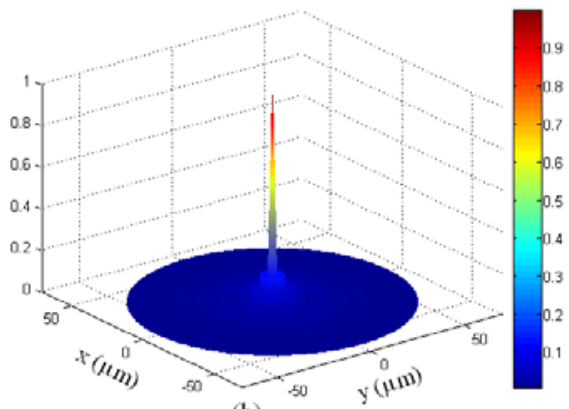

(b)

Fig. 2 Excited field of (a) first and (b) $15^{\text {th }}$ order cladding modes within SCSMF

The simulated spectral response of the SMF28-SCSMF-SMF28 structure is shown in figure 3. In the simulation, we assume the SMF28 has a core diameter of $8.3 \mu \mathrm{m}$, refractive indices of the core and cladding are 1.4504 and 1.4447 respectively and the SCSMF has a length of $40 \mathrm{~mm}$. The loss response displays distinct spectral peaks due to Multimode interferenceMMH.

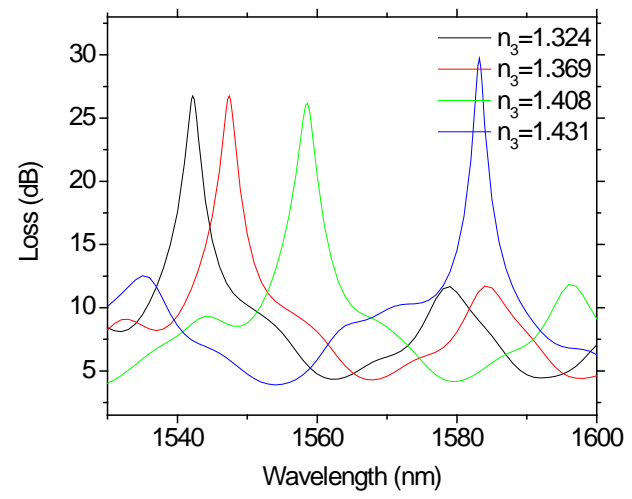

Fig. 3 Spectral response of the refractometer for surrounding liquids with various refractive indices

Figure 3 shows that as the RI increases, the central wavelength of the spectral peak increases. Further simulations of central wavelength shift vs. SRI at different core diameters of 1.6, 2.2 and $2.8 \mu \mathrm{m}$ for SCSMF were carried and the results are shown in Fig. 4. In all these simulations, the fibre parameters are the same as above. 

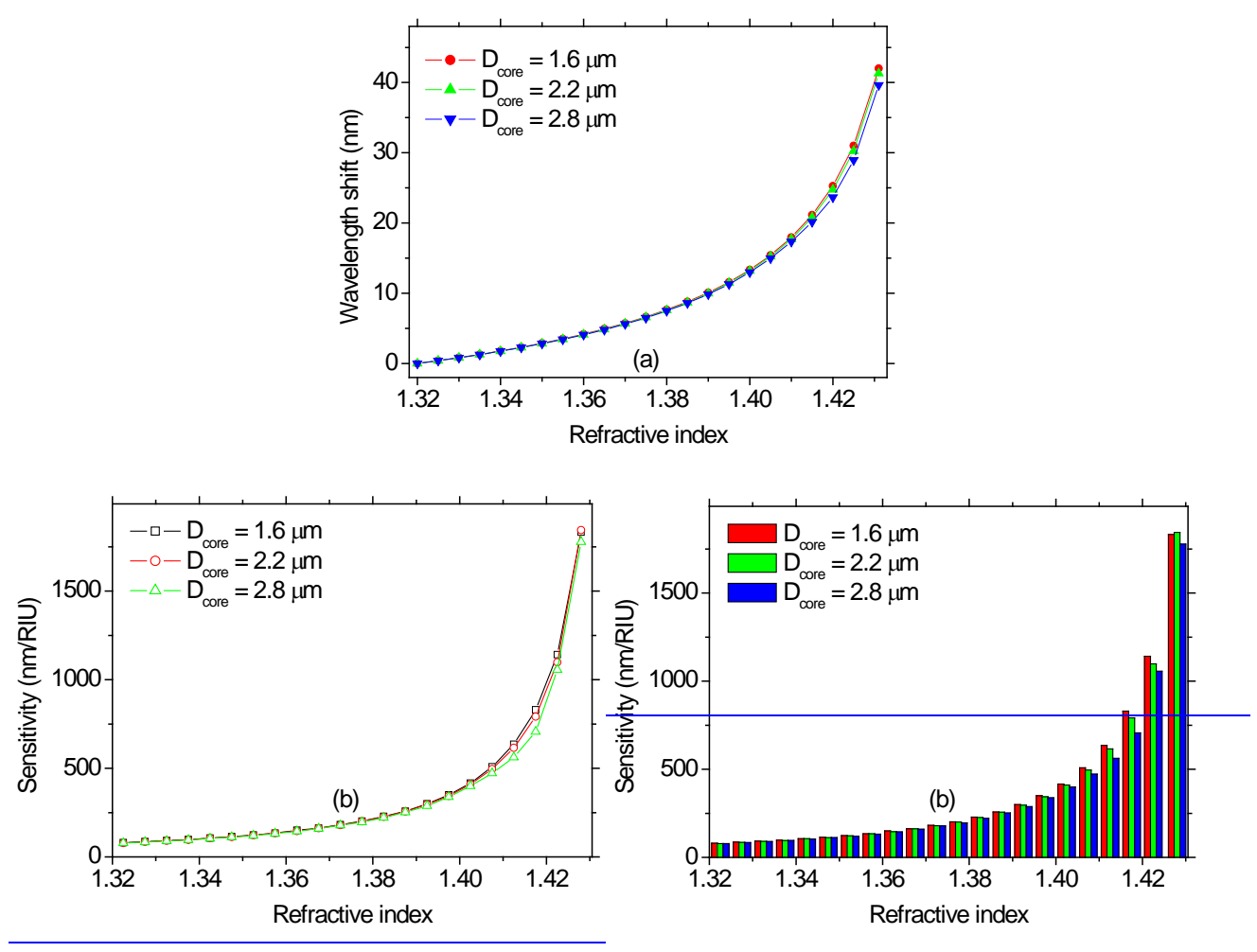

Fig. 4 Calculated (a) central wavelength shift vs. SRI and (b) estimated sensitivity at three different SCSMF core diameters

The simulated results in Figure 4(a) confirm that as SRI increases the central wavelength of the SMF28-SCSMF-SMF28 fiber structure increases monotonically and obeys an exponential curve. The rate of the increase at lower SRI is less than that at higher SRI. It can also be seen from figure 4(a) that the core diameter of SCSMF has only a marginal influence on the wavelength shift when it changed from 1.6 to $2.8 \mu \mathrm{m}$. Figure 4(b) shows that the minimum sensitivities for all the three refractometers with three different SCSMF core diameters are nearly the same and vary from 78 to $80 \mathrm{~nm} / \mathrm{RIU}$ in the SRI range from 1.32 to 1.325 and the maximum sensitivities for the three refractometers vary from 1778 to $1845 \mathrm{~nm} / \mathrm{RIU}$ in the SRI range from 1.425 to 1.431 . The refractometer with a smaller SCSMF core diameter has a slightly higher sensitivity than that with a larger SCSMF core diameter but the difference is not significant.

Another important factor for this type of refractometer is the cladding diameter of an SCSMF. The relationship between wavelength shift and SRI was calculated at different SCSMF cladding diameters but at a fixed SCSMF core diameter of 2.2 
$\mu \mathrm{m}$. The simulation results of central wavelength shift vs. SRI at different SCSMF cladding diameters of 100, 120 and $125 \mu \mathrm{m}$ are shown in Fig. 5.
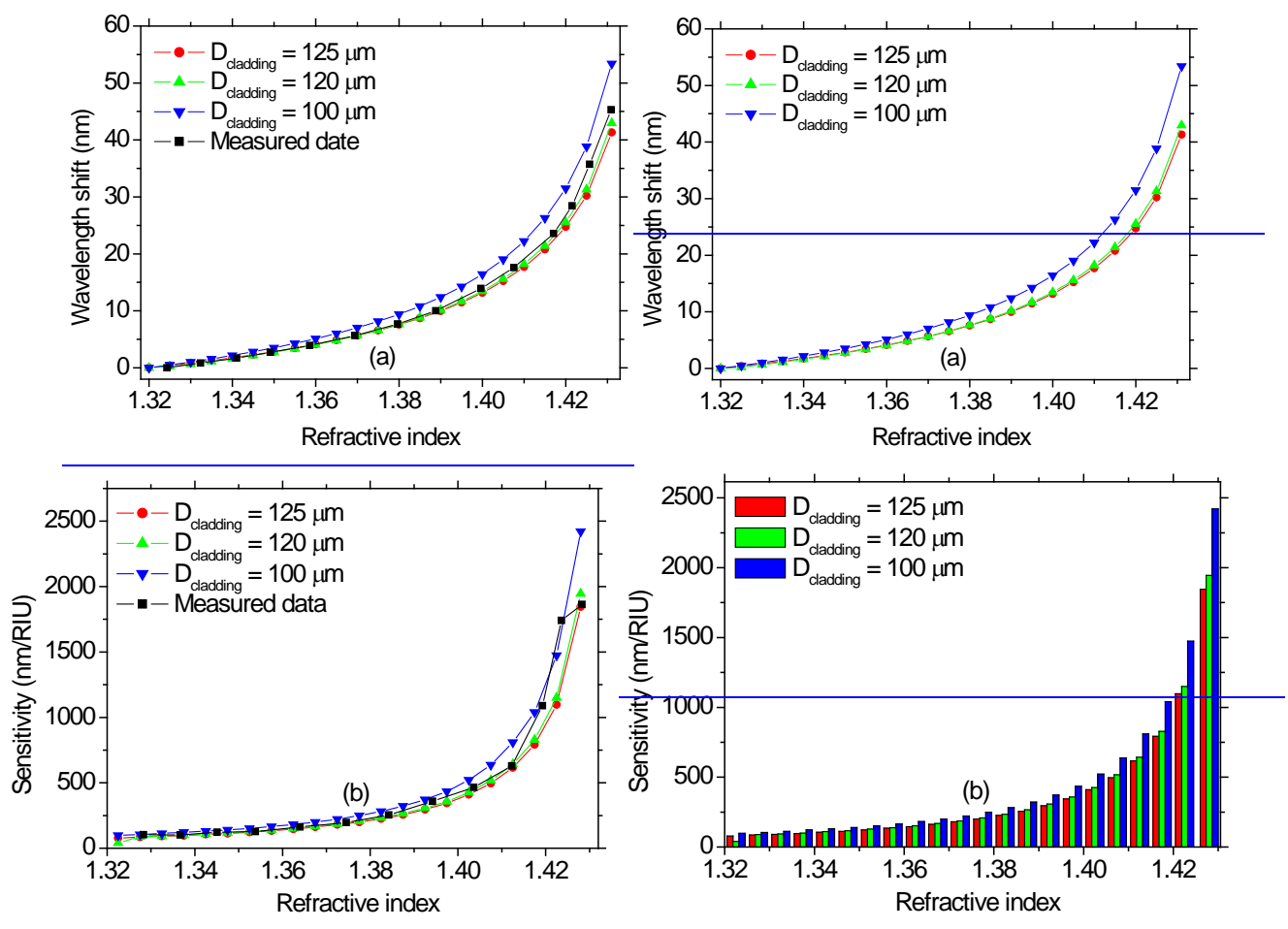

Fig. 5 Calculated and measured (a) central wavelength shift vs. SRI and (b) estimated sensitivity at three different SCSMF eladding diameters

Figure 5 shows that for the same SRI range, a refractometer with smaller cladding diameter of $100 \mu \mathrm{m}$ has larger wavelength shift range and higher sensitivity compared to that with larger cladding diameters. For a commercial optical fiber with cladding diameter of $125 \mu \mathrm{m}$, the typical cladding diameter fabrication error is $\pm 1 \mu \mathrm{m}$ which will not have significant influence on the sensitivity of the refractometer as shown in figure 5(b). However figure 5(b) also indicates that in order to achieve a higher sensitivity, an effective approach is to employ an SCSMF with a smaller cladding diameter.

\section{EXPERIMENTAL INVESTIGATION}

Experimental verification was carried out using the fiber structure above. The SMF28 fiber is a conventional fiber used in optical communications and supplied by Corning. The SCSMF fiber used in our experiments has a cutoff wavelength of 430 
$\mathrm{nm}$ and a length of $40 \mathrm{~mm}$. The SMF28-SCSMF-SMF28 fiber structure was fabricated by a normal automated fusion splicing process. Figure 6 gives a microscopic image of the splice joint between the SCSMF and SMF28 and its spectral response at different SRIs.
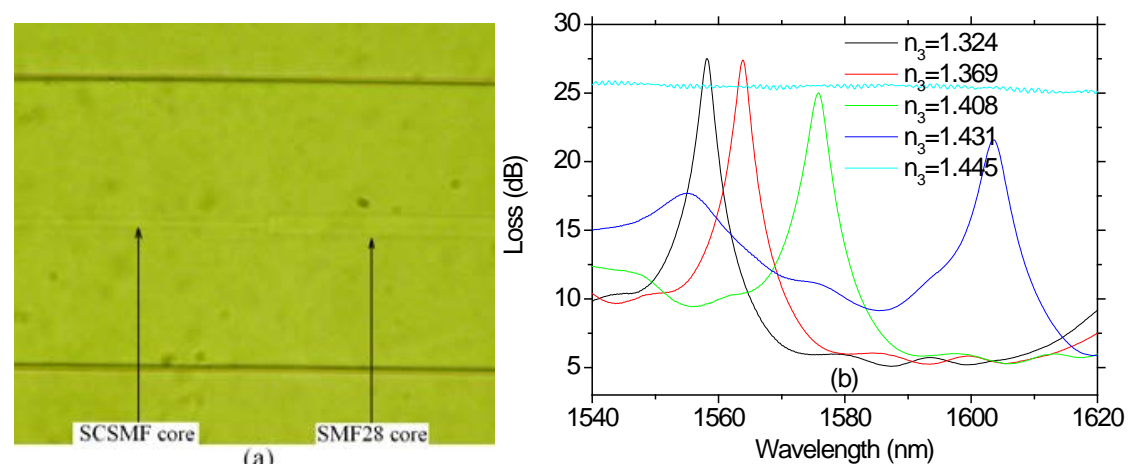

Fig. 6 (a) A microscopic image of the splice joint between the SMF28 and SCSMF and (b) measured spectral response of this structure at different SRIs

Figure 6(a) shows that the splice between SMF28 and SCSMF was well aligned and visually shows that the core diameter of SCSMF is much smaller than that of SMF28. Figure 6(b) shows that as the SRI increases, the central wavelength of the loss peak increases, confirming our theoretical predictions as shown in figure 3. When the SRI increases to a value close to the RI of the cladding, no cladding modes are supported and the only possible is the core mode, if it exists. Our measured result shows that the transmission loss in the SMF28-SCSMF-SMF28 structure is circa $25 \mathrm{~dB}$, which confirms the theoretical assumption made earlier that there is effectively no guided mode within the SCSMF core and that at values of the SRI close to the RI of the cladding, the cladding mode is also suppressed. The wavelength shifts vs. different SRIs and the estimated sensitivity of the central wavelength to the change in SRI are shown in figure $7 \underline{5}$. It is noted that a $3 \mathrm{~dB}$ mean wavelength is used as an estimate of the measured peak wavelength in this paper because it is a more reliable measurement than the actual peak wavelength. 

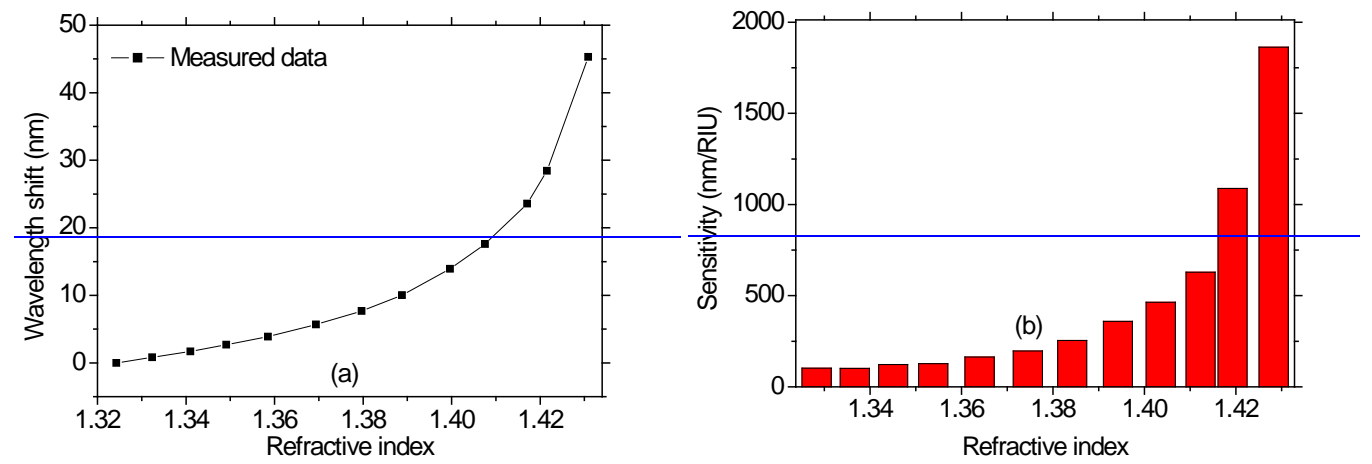

Fig. 7 (a) measured spectral response shifts vs. SRI and (b) estimated sensitivity

Figure 7ㅁa) shows that as the SRI increases the wavelength shift increases monotonically but that the slope of the increase is the highest at higher liquid refractive indices. Therefore, it can be seen from Figure $7 \underline{5}$ (b), that as expected the SRI sensitivity in the RI range from 1.422 to 1.431 is larger than that in the lower RI range. The maximum and minimum sensitivities of this refractometer in the RI range from 1.324 to 1.431 are 102 and $1808 \mathrm{~nm} / \mathrm{RIU}$ respectively. Comparing the simulated and measured results in figure 7 w figure 4 , it is easy to conclude that the experimental results agree well with the simulation results. It is worth noting that the measured wavelength shift vs. RI in [21-22] has linear or quasi-linear response instead of exponential. This is due to the fact that the SRI measurement range used in [21-22] is from 1.33 to 1.40 which has a quasi-linear response at lower RI range as shown in figure $7 \underline{5}$ (a). As the SRI increases to a value close to that of the RI of the fiber cladding, the response becomes strongly nonlinear which we have verified by both simulation results and experimental results in this paper.

\section{CONCLUSION}

In conclusion a comprehensive theoretical model for an SMF28-SCSMF-SMF28 structure based refractometer is developed based on MPA method. Simulation results show that the wavelength shift undergoes an exponential increase as the SRI increases and importantly that the core diameter of the SCSMF used does not significantly influence the sensitivity of the refractometer but that the cladding diameter of the SCSMF does influence sensitivity - an SCSMF with smaller cladding can improve the RI sensitivity. An experimental investigation confirms the validity of the simulation and demonstrates that it is 


\section{ACKNOWLEDGEMENT}

Qiang Wu is funded by Science Foundation Ireland under grant no. 07/SK/I1200. Pengfei Wang is funded by the Irish Research Council for Science, Engineering and Technology, and co-funded by the Marie-Curie Actions under FP7.

\section{REFERENCES}

[1]. M. Han, F. W. Guo, Y. F. Lu, “Optical fiber refractometer based on cladding-mode Bragg grating”, Optics Letters, vol. 35, no. 3, pp. 399-401, 2010

[2]. T. Guo, H. Y. Tam, P. A. Krug, J. Albert, "Reflective tilted fiber Bragg grating refractometer based on strong cladding to core recoupling”, Optics Express, vol. 17, no. 7, pp. 5736-5742, 2009

[3]. O. Frazao, T. Martynkien, J. M. Baptista, J. L. Santos, W. Urbanczyk, J. Wojcik, “Optical refractometer based on a birefringent Bragg grating written in an H-shaped fiber”, Optics Letters, vol. 34, no. 1, pp. 76-78, 2009

[4]. H. Tsuda, K. Urabe, “Characterization of long-period grating refractive index sensors and their applications”, Sensors, vol. 9, no. 6, pp. 4559-4571, 2009

[5]. J. H. Chong, P. Shum, H. Haryono, A. Yohana, M. K. Rao, C. Lu and Y. Zhu, "Measurements of refractive index sensitivity using long-period grating refractometer”, Opt. Commun. 229 (2004), pp. 65-69

[6]. J. Yang, L. Yang, C.Q. Xu, C. Xu, W. Huang and Y. Li, "Long-period grating refractive index sensor with a modified cladding structure for large operational range and high sensitivity”, Appl. Opt. 45 (2006), pp. 6142-6147

[7]. H. M. Liang, H. Miranto, N. Granqvist, J. W. Sadowski, T. Viitala, B. C. Wang, M . Yliperttula, "Surface Plasmon resonance instrument as s refractometer for liquids and ultrathin films”, Senosrs and Actuators B-Chemical, vol. 149, no. 1, pp. 212-220, 2010

[8]. J. Y. Lee, S. K. Tsai, "Measurement of refractive index variation of liquids by surface Plasmon resonance and wavelength-modulated heterodyne interferometry", Optics Communications, vol. 284, no. 4, pp. 925-929, 2011

[9]. J. Castillo, H. Gutierrez, J. Chirinos, J. C. Perez, "Surface plasmon resonance device with imaging processing detector for refractive index measurements”, Optics Communicatins, vol. 283, no. 20, pp. 3926-3930, 2010

[10]. M. I. Zibaii, A. kazemi, H. Latifi, M. K. Azar, S. M. Hosseini, M. H. Ghezelaiagh, "Measuring bacterial growth by refractive index tapered fiber optic biosensor”, Journal of Photochemistry and Photobiology B-Biology, vol. 101, no. 3, pp. 301-320, 2010

[11]. Y. H. Tai, P. K. Wei, “Sensitive liquid refractive index sensors using tapered optical fiber tips”, Optics Letters, vol. 35, no. 7, pp. 944-946, 2010

[12]. J. Arrue, F. Jimenez, G. Aldabaldetreku, G. Durana, J. Zubia, M. Lomer, J. Mateo, “Analysis of the use of tapered graded-index polymer optical fibers for refractive-index sensors”, Optics Express, vol. 16, no. 21, pp. 16616-16631, 2008

[13]. Q. Wu, Y. Semenova, P. Wang and G. Farrell, "High sensitivity SMS fiber structure based refractometer - analysis and experiment”, Optics Express, vol. 19, no. 9, pp. 7937-7944, 2011

[14]. Q. Wu, Y. Semenova, B. B. Yan, Y. Q. Ma, P. Wang, C. X. Yu and G. Farrell, "Fiber refractometer based on a fiber Bragg grating and single-mode-multimode-single-mode fiber structure”, Optics Letters, vol. 36, no. 12, pp. 2197-2199, 2011

[15]. L. B. Soldano, E. C. M. Pennings, “Optical multi-mode interference devices based on self-imaging: principles and applications”, Journal of Lightwave Technology, vol. 13, no. 4, pp. 615-627, 1995

[16]. W. S. Mohammed, A. Mehta and E. G. Johnson, "Wavelength tunable fiber lens based on multimode interference”, Journal of Lightwave Technology, 
vol. 22, no. 2, pp. 469-477, 2004

[17]. Q. Wu, A. M. Hatta, P. Wang, Y. Semenova and G. Farrell, "Use of a Bent Single SMS Fiber Structure for Simultaneous Measurement of Displacement and Temperature Sensing”, IEEE Photon. Tech. Lett., vol. 23, no. 2, pp. 130-132, 2011

[18]. Q. Wu, Y. Semenova, P. Wang and G. Farrell, "Fibre heterostructure for simultaneous strain and temperature measurement”, Electronics Letters, vol. 47, no. 12, 713-714, 2011

[19]. S. M. Tripathi, A. Kumar, R. K. Varshney, et al., "Strain and Temperature Sensing Characteristics of Single-Mode-Multimode-Single-Mode Structures”, Journal of Lightwave Technology, vol. 27, no. 13, pp. 2348-2356, 2009

[20]. Q. Wu, A. M. Hatta, Y. Semenova and G. Farrell, "Use of a SMS fiber filter for interrogating FBG strain sensors with dynamic temperature compensation”, Applied Optics, vol. 48, pp. 5451-5458, 2009

[21]. T. H. Xia, A. P. Zhang, B. B. Gu, J. J. Zhu, "Fiber-optic refractive-index sensors based on transmissive and reflective thin-core fiber modal interferometers”, Optics Communications, 283 (2010) 2136-2139

[22]. B. Gu, M. J. Yin, A. P. Zhang, J. W. Qian and S. He, “Optical fiber relative humidity sensor based on FBG incorporated thin-core fiber modal interferometer”, Optics Express, vol. 19, no. 5, pp. 4140-4146, 2011

[23]. Q. Wu, Y. Semenova, J. Mathew, P. Wang and G. Farrell, "Humidity sensor based on a singlemode hetero-core fiber structure”, Optics Letters, vol. 36, no, 10, pp. 1752-1754, 2011

[24]. A. W. Snyder and J. D. Love, Optical Waveguide Theory. London: Chapman and Hall, 1983

[25]. L. C. Bobb, P. M. Shankar, H. D. Krumboltz, “Bending effects in biconically tapered single-mode fibers”, Journal of Lightwave Technol., vol. 8, no. 7, pp. 1084-1090, 1990

[26]. M. Monerie, "Propagation in doubly clad single-mode fibers”, IEEE Journal of quantum electronics, vol. QE-18, no. 4, pp. 535-542, 1982

[27]. H. G. Unger, Planar optical waveguides and fibres, Oxford, England: Clarendon, pp. 312-313, 1977

[28]. Y. H. Kim, M. J. Kim, B. S. Rho, M. S. Park, J. H. Jang, and B. H. Lee, "Ultra sensitive fiber-optic hydrogen sensor based on high order cladding mode,” IEEE Sensors Journal, vol.. 11, no, 6, pp.1423-1426, 2011. 